\title{
EIF3G is associated with narcolepsy across ethnicities
}

\author{
Anja Holm ${ }^{1,2}$, Ling Lin ${ }^{3}$, Juliette Faraco ${ }^{3}$, Sara Mostafavi ${ }^{4}$, Alexis Battle ${ }^{4}$, Xiaowei Zhu ${ }^{5}$, Douglas F Levinson ${ }^{5}$, \\ Fang $\mathrm{Han}^{6}$, Steen Gammeltoft ${ }^{1}$, Poul Jennum ${ }^{2}$, Emmanuel Mignot ${ }^{3}$ and Birgitte R Kornum ${ }^{\star, 1}$
}

Type 1 narcolepsy, an autoimmune disease affecting hypocretin (orexin) neurons, is strongly associated with HLA-DQB1*06:02. Among polymorphisms associated with the disease is single-nucleotide polymorphism rs 2305795 (c. ${ }^{*} 638 \mathrm{G}>\mathrm{A}$ ) located within the $P 2 R Y 11$ gene. $P 2 R Y 11$ is in a region of synteny conserved in mammals and zebrafish containing PPAN, EIF3G and DNMT1 (DNA methyltransferase 1). As mutations in DNMT1 cause a rare dominant form of narcolepsy in association with deafness, cerebellar ataxia and dementia, we questioned whether the association with $P 2 R Y 11$ in sporadic narcolepsy could be secondary to linkage disequilibrium with DNMT1. Based on genome-wide association data from two cohorts of European and Chinese ancestry, we found that the narcolepsy association signal drops sharply between P2RY11/EIF3G and DNMT1, suggesting that the association with narcolepsy does not extend into the DNMT1 gene region. Interestingly, using transethnic mapping, we identified a novel single-nucleotide polymorphism rs3826784 (c.596-260A $>$ G) in the EIF3G gene also associated with narcolepsy. The disease-associated allele increases EIF3G mRNA expression. EIF3G is located in the narcolepsy risk locus and EIF3G expression correlates with PPAN and P2RY11 expression. This suggests shared regulatory mechanisms that might be affected by the polymorphism and are of relevance to narcolepsy.

European Journal of Human Genetics (2015) 23, 1573-1580; doi:10.1038/ejhg.2015.4; published online 11 February 2015

\section{INTRODUCTION}

Narcolepsy with cataplexy (Type 1 narcolepsy) affects 1 in 3000 individuals and is caused by the loss of around 70000 hypocretin(hcrt, also known as orexin) producing neurons in the hypothalamus. The disease is strongly associated with HLA-DQB1 ${ }^{\star} 06: 02$ and DQA1 ${ }^{\star} 01: 02,{ }^{1}$ the T-cell receptor alpha locus ${ }^{2}$ and polymorphisms in other immune-related genes. ${ }^{3}$ The loss of hcrt-producing cells is hypothesized to have an autoimmune basis. ${ }^{4}$

One of the genetic associations in narcolepsy is rs2305795, a polymorphism located in the purinergic receptor $P 2 R Y 11^{5,6}$ The disease-associated allele of rs 2305795 decreases P2RY11 expression and increases the sensitivity of $\mathrm{CD}^{+} \mathrm{T}$ cells to cell death induced by ATP. Interestingly, we also recently found that missense mutation in exon 21 of the DNA methyltransferase 1 (DNMT1) gene causes a rare hereditary form of narcolepsy-associated deafness, cerebellar ataxia and eventually dementia (ADCA-DN). ${ }^{7}$ As the P2RY11 and DNMT1 genes are located in close proximity (within $18 \mathrm{~kb}$ ) on chromosome 19, we hypothesized that the two signals could be related at the pathophysiological level. In support of this hypothesis, Kornum et al. ${ }^{5}$ observed a correlation between P2RY11 and DNMT1 expression in peripheral blood mononuclear cells (PBMCs) of both patients and healthy controls. These findings made us question whether the association with P2RY11 in spontaneous narcolepsy could be secondary to linkage disequilibrium (LD) with DNMT1. In the original study, Kornum et al..$^{5}$ fine-mapped the locus using seven single-nucleotide polymorphisms (SNPs) and reported an association between DNMT1 and narcolepsy in individuals of European ancestry but not Asians. In Asians, LD pattern pointed towards an association with P2RY11. ${ }^{5}$
One of the biggest challenges of association studies is to identify which genetic variant is causal within a given locus. To address this challenge, increased sample size, functional studies and transethnic mapping may be beneficial. In transethnic mapping, different LD patterns in the disease-associated locus can be explored to narrow down the associated genetic region. This approach has previously been shown to be very powerful in narcolepsy and other diseases. ${ }^{1,8,9}$ In this study, we reinvestigated SNPs in the P2RY11-DNMT1 region in narcolepsy across three different ethnic backgrounds. Based on genome-wide association study (GWAS) data available in Chinese and Caucasians, we observed that the narcolepsy association signal drops sharply between P2RY11 and DNMT1 in both cohorts. Based on LD patterns, we next selected four SNPs for genotyping in a cohort of African Americans. Following genotyping, we identified a novel SNP in EIF3G, rs3826784, best associated with narcolepsy across all three cohorts. EIF3G is located between P2RY11 and DNMT1. To further explore association between these different genes and narcolepsy, we finally also examined gene expression patterns in the entire region.

\section{MATERIALS AND METHODS}

Subjects

Narcolepsy with cataplexy cases were selected based on documented hypocretin deficiency or clearcut cataplexy and HLA-DQB1 ${ }^{\star} 06: 02 .^{2,6}$ The initial European ancestry GWA data set was comprised of 807 cases and 1074 DQB1 ${ }^{\star 06: 02-}$ positive controls: 415 cases and 753 controls were recruited from the United States and Canada; 392 cases and 321 controls were recruited from European centers. The Chinese GWA data set was comprised of 1078 cases and 1903 controls. Analysis of the GWA data (549596 SNPs in Europeans and 603382 SNPs in Chinese) was performed as described. ${ }^{2,6}$ The African-American sample

\footnotetext{
${ }^{1}$ Molecular Sleep Laboratory, Department of Diagnostics, Glostrup University Hospital, Glostrup, Denmark; ${ }^{2}$ Danish Center for Sleep Medicine, Department of Neurophysiology, Glostrup Hospital, University of Copenhagen, Glostrup, Denmark; ${ }^{3}$ Center for Sleep Sciences in Medicine and Department of Psychiatry, Stanford University School of Medicine, Palo Alto, CA, USA; ${ }^{4}$ Department of Computer Science, Stanford University, Palo Alto, CA, USA; ${ }^{5}$ Department of Psychiatry and Behavioral Sciences, Stanford University, Palo Alto, CA, USA; ${ }^{6}$ Department of Pulmonary, Critical Care Medicine, Peking University People's Hospital, Beijing, China

*Correspondence: Dr BR Kornum, Molecular Sleep Laboratory, Department of Diagnostics, Glostrup University Hospital, Nordre Ringvej 69, Glostrup 2600, Denmark. Tel: +45 38634541; Fax: +45 38633958; E-mail: birgitte.kornum@regionh.dk

Received 26 February 2014; revised 28 October 2014; accepted 9 December 2014; published online 11 February 2015
} 
contained 1297 individuals (249 cases, 1048 controls). The controls were recruited from the National Institute of Mental Health (NIMH).

For the expression quantitative trait locus (eQTL) analysis, we used a large case-control study of major depression, for which RNA-sequencing data from whole blood together with genotype data were available for 922 individuals of European ancestry.

\section{Imputation of Chinese and European cohorts}

Imputation for the PPAN-DNMT1 region (from rs7250025 to rs2043305) was performed in the European and Chinese cohorts separately, using Beagle v.3.2. $1^{10}$ against reference genotypes from the 1000 genomes integrated data set. Chinese genotypes, originally obtained through the Affymetrix Axiom CHP array, were imputed against the $\mathrm{CHB}$ reference population. European ancestry genotypes from the Affymetrix 6.0 array were imputed using four European populations as a reference (CEU, TSI, GBR, IBS). In the paper by Kornum et al., ${ }^{5}$ SNPs rs2305795 and rs12460842 were fine typed in many of the Caucasian samples. Further, rs2305795 was also retyped in the Chinese cohort ${ }^{6}$ and all the results were consistent with imputed results. The imputation quality score for rs 3826784 was consistently above 0.8 and the overall association on pooled imputed genotypes is considered valid. Therefore, SNP markers with poor imputation quality score in either Chinese or Europeans $\left(r^{2}<0.8\right)$ were excluded from all further analysis.

The data of this study is submitted to the ClinVar Database (http://www.ncbi. nlm.nih.gov/clinvar/; accession numbers: SCV000196702-SCV000196705).

\section{Fine mapping of the chromosome 19 locus, and replication of published SNPs}

We used 1297 African-American individuals (249 cases, 1048 controls) for genotyping in the region of association. Based on LD and $r^{2}$ data from the International HapMap Project (Hapmap.org), we observed that the LD pattern between the SNPs found from the GWAS was different in African Americans.

The selected SNPs were rs1551570: NM_020230.5:c.190-151C>T; rs12460842: NM_002566.4:c. - 203A > G; rs2305795: NM_002566.4:c. ${ }^{*} 638 \mathrm{G}>$ A; and rs3826784: NM_003755.3:c.596-260A>G. For genotyping, we used predesigned Taqman SNP genotyping assays (Applied Biosystems, Carlsbad, CA, USA) to validate the observed LD pattern from the International HapMap project in a cohort of African-American narcolepsy patients and controls. Genotyping was performed at Stanford University. SNPs rs1551750, rs12460842 and rs3826784 are all located within introns of P2RY11 and EIF3G, respectively. SNP rs2305795 is located in the $3^{\prime}$-UTR of P2RY11. Genotype results were analyzed (allelic associations, LD calculations and analysis) and standard $r^{2}$ plots were generated using Haploview 4.2. ${ }^{11}$

\section{RNA sequencing and genotyping of 922 individuals of European ancestry}

The complete procedure for whole blood processing, RNA sequencing and genotyping is described in Battle et al. ${ }^{12}$ Briefly, whole blood was collected in PAXGene tubes for RNA and in acid-citrate-dextrose tubes for DNA. Tubes were stored at $-80^{\circ} \mathrm{C}$. Total RNA was extracted using the GLOBINclear Kit (Invitrogen, Carlsbad, CA, USA). Libraries for RNA sequencing was performed using Illumina TruSeq protocol. DNA was genotyped on the Illumina HumanOmni1-Quad BeadChip (Illumina, San Diego, CA, USA).

\section{Statistical analysis}

Genome-wide association analysis of the European and Chinese ancestry has been described previously. ${ }^{2,6}$ Statistical analysis of genotyped and imputed genotype data were performed using the PLINK software package (v.1.07) (http://pngu.mgh.harvard.edu/purcell/plink/), ${ }^{13}$ and in Haploview 4.2. ${ }^{11}$

Associations within ethnic groups were performed with basic allelic $\chi^{2}$ statistic tests. For associations combining multiple ethnic groups, the MantelHaenszel test was used together with the Breslow Day test of homogeneity of the odds ratio (both implemented in Plink).

The details of the cis-eQTL analysis are described in Battle et al. ${ }^{12}$ Briefly, expression data was first normalized to remove the effect of technical factors, population structure and 'hidden' factors (which have been shown to reduce the statistical power to identify cis-eQTLs $\left.{ }^{14}\right)$. Such normalization approaches can significantly increase power for detecting cis-eQTLs; however, in doing so, such approaches also remove broader expression patterns that may be driven by trans-regulation (as a trans-acting factor may affect the expression levels of multiple target genes) or reflect coregulation. As a result, previous work has shown that separate normalization of gene expression data may be appropriate for detecting cis versus trans associations. ${ }^{12,14}$ Therefore, we quantified coexpression levels between genes using the normalization approach optimized for detecting trans-eQTLs. For each SNP, a $P$-value for association with gene expression level of each nearby gene (1 Mb from the transcription start site) was computed based on the Spearman's correlation coefficient. Significant associations were reported using 0.05 FDR (false discovery rate).

PPAN: NM_020230.4; P2RY11: NM_002566.4; EIF3G: NM_003755.3; DNMT1: NM_001130823.1.

Rs1551570: NM_020230.5:c.190-151C>T; rs12460842: NM_002566.4:c.

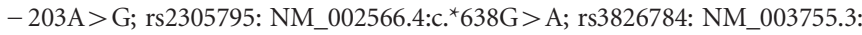
c. $596-260 \mathrm{~A}>\mathrm{G}$.

SNPs reported in Table 1: rs1551570: NM_020230.5:c.190-151C>T; rs12460842: NM_002566.4:c. $-203 A>$ G; $\quad$ rs2010353: NM_002566.4:c.19 $+142 \mathrm{G}>\mathrm{T}$; rs3826784: NM_003755.3:c.596-260T >C; rs2305795: NM_ 002566.4:c. ${ }^{*} 638 \mathrm{G}>$ A; rs12462506: NM_002566.4:c.20 - 178T > G; rs3826785: NM_003755.3:c.595+327G > A; rs73011220: NM_002566.4:c.19+880G > A; rs112647895: NM_002566.4:c.19+446G $>$ A; rs3745601: NM_002566.4: c.259G > A; rs2305789: NM_020230.5:c.291+50A > G; rs6511570: NM_ 001130823.1:c.2895-855C > T; rs2288349: NM_001130823.1:c.2721-45C>T; rs35693490: NM_001130823.1:c.2721 -959G > A; rs8101626: NM_001130823.1: c.4773+383C > T; rs4804122: NC_000019.10:g.10131268C > T; 9 rs2114724: NM_001130823.1:c.1832+14A > G; rs2290684: NM_001130823.1:c.3394 +34T>C; rs8112801: NM_001130823.1:c.3117-203A > C; rs11880553: NM_001130823.1:c.3117-660G > A; rs11880388: NM_001130823.1: c.3117-677C> T; rs11085587: NC_000019.10:g.10124423G >C; rs35374357: NC_000019.10:g.10129267T >C; rs7710: NM_003755.3:c.846T >C; rs870612: NC_000019.10:g.10120814A > C; rs4555265: NC_000019.10:g.10121508G > A; rs11667630: NM_003755.3:c.300+47G > T; rs2290687: NM_003755.3:c.240 +23G > A; rs55752217: NM_020230.5:c.292-46C > T; rs2305792: NM_ 020230.5:c.902-10C>G; rs1037686: NM_002566.4:c.19+419T>A; rs3745600: NM_002566.4:c.237C > T; rs34484805: NM_002566.4:c.20 - 882A > G; rs7401: NM_002566.4:c. ${ }^{*} 363 \mathrm{~A}>\mathrm{G}$; rs11666402: NM_020230.5:c.513+266T > C; rs10414661: NC_000019.10:g.10131561T >A; rs12462004: NC_000019.10: g.10127588T > G; rs10404209: NC_000019.10:g.10126751T >C; rs9305012: NM_001130823.1:c.2266-225A > G; rs10407514: NM_001130823.1: c.2895-449G >C; rs10418707: NM_001130823.1:c.2894+810C> T; rs12611113: NM_001130823.1:c.2721-212A > G; rs60565702: NM_001130823.1:c.2720 +999C > T; rs72620548: NM_001130823.1:c.4490-306C > T; rs10854076: NM_001130823.1:c.4293+238C > G; rs4804489: NM_001130823.1:c.2265 +418T > C; $\quad$ rs57366074: NM_001130823.1:c.2586+99G > T; $\quad$ rs10414537: NC_000019.10:g.10126048C > T.

\section{RESULTS}

\section{Association signal within the risk locus}

Using two GWA data sets from narcoleptic patients and controls of European and Chinese ancestry, we investigated polymorphisms in the P2RY11-DNMT1 region. The cohorts included 1881 individuals of European ancestry (807 cases and 1074 controls) and 2981 Chinese (1078 cases and 1903 controls). SNPs in the region of interest were imputed in each GWA data set, and ranked by statistical significance in the Chinese cohort (Table 1). The most significant SNPs in both GWA data sets are in high LD with rs2305795 and located in the PPAN-P2RY11-EIF3G haplotype block, and not in DNMT1 (Table 1). $R^{2}$ plots clearly illustrate that SNPs in high LD with rs2305795 are restricted to the PPAN-P2RY11-EIF3G region, but not DNMT1 (Figure 1), as was also observed by Han et al. ${ }^{6}$ Based on this analysis, five SNP markers (rs1551570 (PPAN), rs12460842 (P2RY11), rs2010353 (P2RY11), rs2305795 (P2RY11) and rs3826784 (EIF3G) 


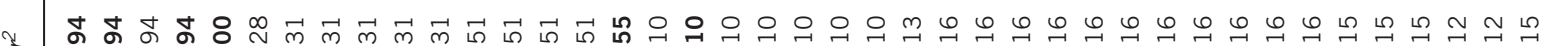

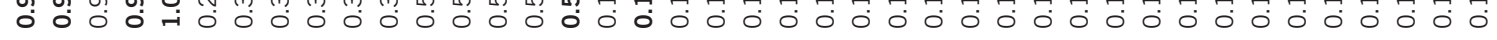

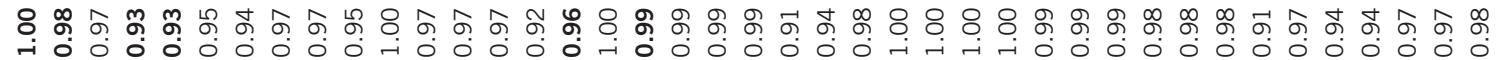

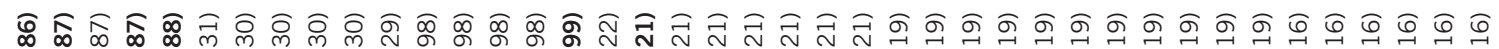

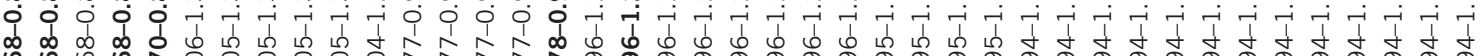

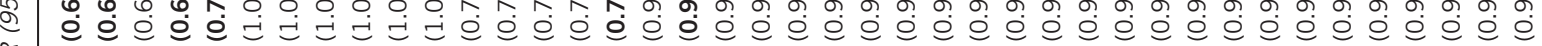

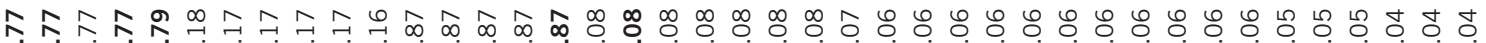

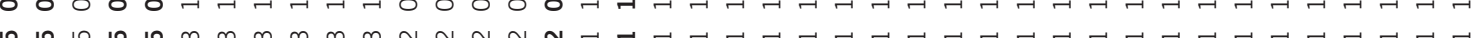

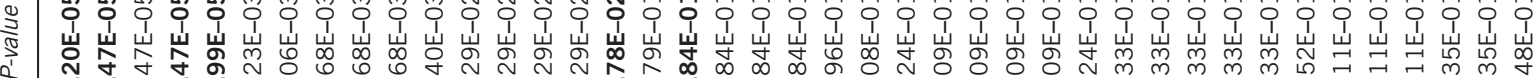

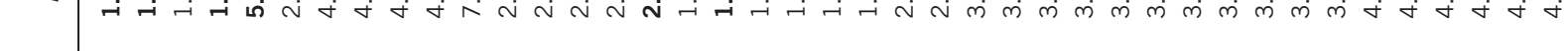

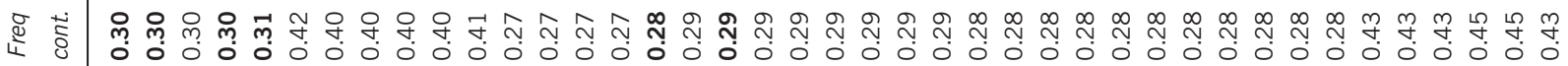

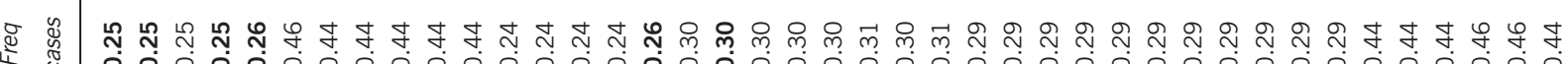

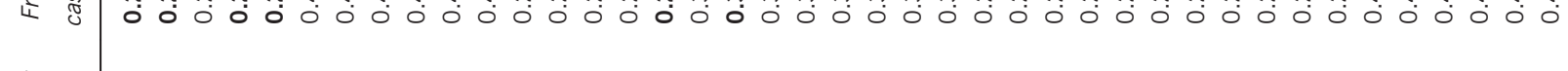

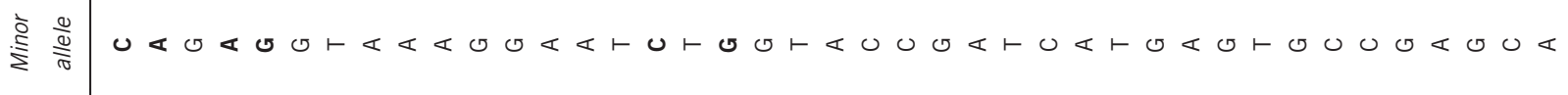

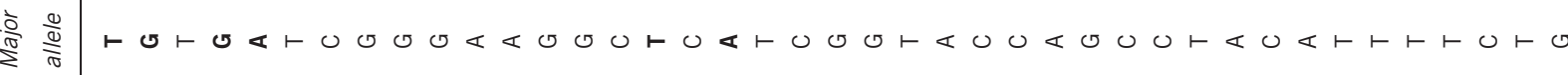
용

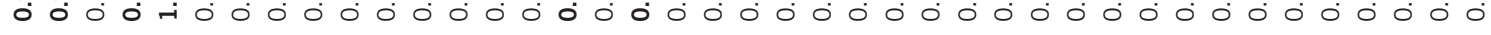

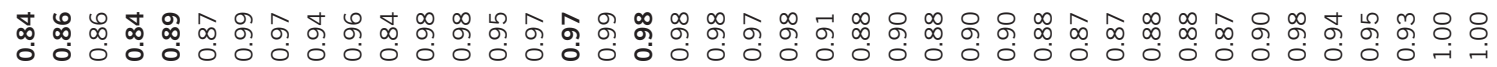
১ চ

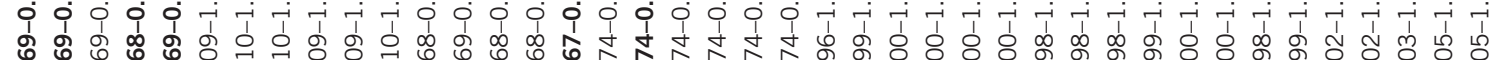

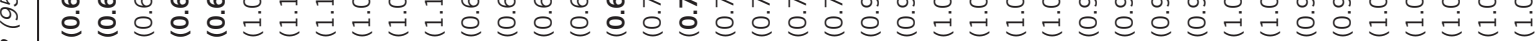
옷 オ

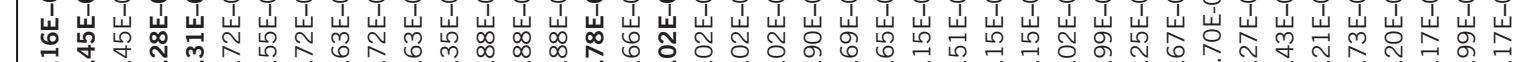

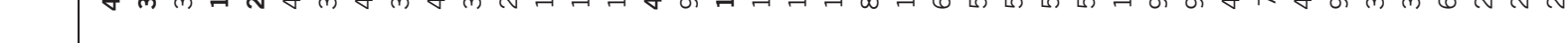

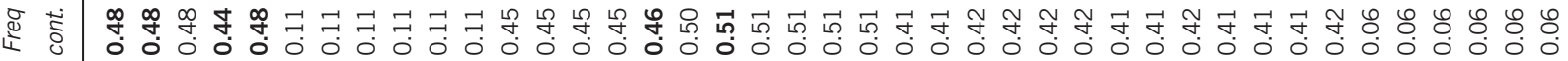
ฮ

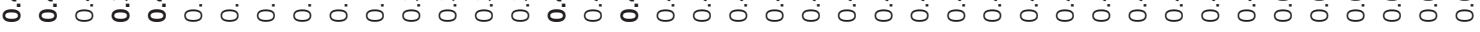
(1)

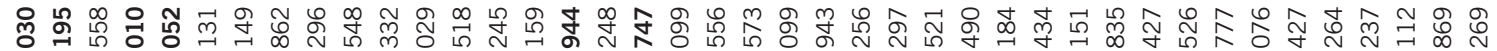
क

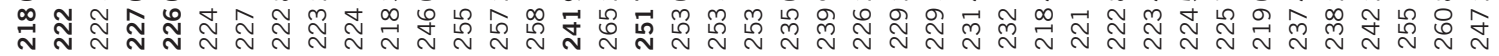

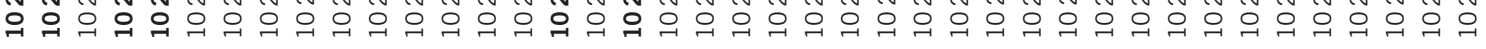

우

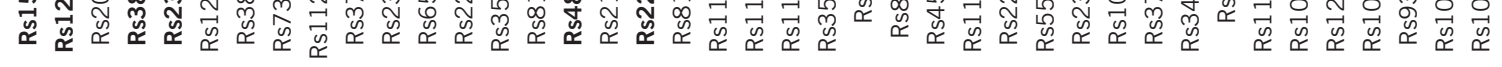

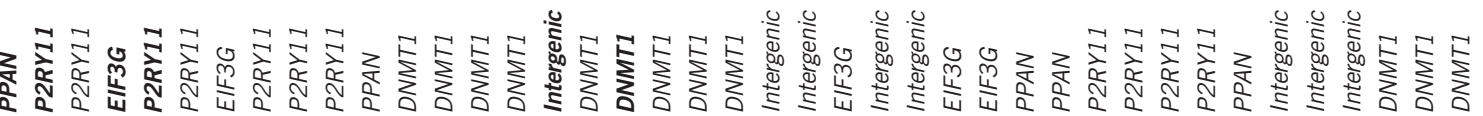


were significantly associated with narcolepsy in both cohorts, and were selected for further analysis. Owing to a failure of the genotyping assay, however, rs2010353 was excluded, leaving four SNPs for all subsequent analyses.

Based on data from the International HapMap Project, we next explored LD patterns for the four markers across ethnic groups. Interestingly, two SNPs rs12460842 (P2RY11) and rs3826784 (EIF3G) had a lower LD with rs1551570 (PPAN) and rs2305795 (P2RY11) in an African-American cohort (Figure 2), suggesting these to be candidates for transethnic mapping. Genotyping of these four markers in 1297 African Americans (249 cases and 1048 controls) showed that surprisingly rs3826784, and not the previously reported rs2305795, showed the strongest narcolepsy association based on odds ratio (OR) values, although none of the four SNPs reached statistical significance in this data set. A meta-analysis was performed across all ethnic groups and here we replicated the finding by Kornum et al. ${ }^{5}$ of an increased OR with the major allele rs2305795A (Table 2 shows data on minor allele). The meta-analysis further revealed that rs3826784 showed the strongest association with narcolepsy across all ethnic groups (Table 2) with a slightly higher OR for the rs3826784G risk allele and a lower $P$-value. There were no significant differences between the ORs from the four SNPs.

\section{Expression quantitative trait loci analysis}

To determine if rs3826784 regulates gene expression, we next performed an eQTL analysis of the genes in the risk locus. For this analysis, we used a large case-control study of major depression, for which RNA-sequencing data from whole blood together with genotype data were available for 922 individuals of European ancestry. ${ }^{12}$ After appropriate normalization, whether or not cases or controls were used it did not influence the eQTL analysis; ${ }^{12}$ thus, we performed all analysis in the combined sample.

Using this data set, we found that the expression of EIF3G increased with the risk allele, DNMT1 expression decreased and the expression of PPAN and P2RY11 did not change with the risk allele (rs3826784G) (Figure 3). Furthermore, the expression of EIF3G and DNMT1, but not PPAN and P2RY11, correlated with rs3826784, the strongest association being with EIF3G (Table 3). The other four SNPs in our analysis showed equally strong association with EIF3G consistent with the high LD between these four SNPs in Caucasians (Figure 2). The expression of DNMT1 was most strongly correlated with eQTL SNP for DNMT1 rs2290684 (highlighted in Table 1). The effect of rs3826784 on DNMT1 expression is therefore likely explained by LD between $\mathrm{rs} 3826784$ and $\mathrm{rs} 2290684\left(r^{2}=0.35\right)$. In line with this, conditional analysis showed that rs3826784 does not independently affect DNMT1 expression when the effect of rs2290684 is controlled for $(P>0.1)$.

The effect of rs 2305795 on P2RY11 was also re-examined. Using our data, we found that rs2305795 was not an eQTL for P2RY11 (see Supplementary Table S1). In addition, as rs2305795 is located in the 3'-UTR of P2RY11, it was possible to analyze allele-specific association for this SNP. Using a test of association between rs2305795 and the allelic ratio of P2RY11 (as an eQTL), we did not observe a significant association between rs2305795 and the allelic expression of P2RY11 in the human whole blood data set in contrast to earlier reports in PBMCs. ${ }^{5}$

\section{Gene expression correlation}

We finally investigated the possibility of coregulation and/or regulatory relationships between the four genes in our locus by analyzing correlations between expression of PPAN, P2RY11, EIF3G and 


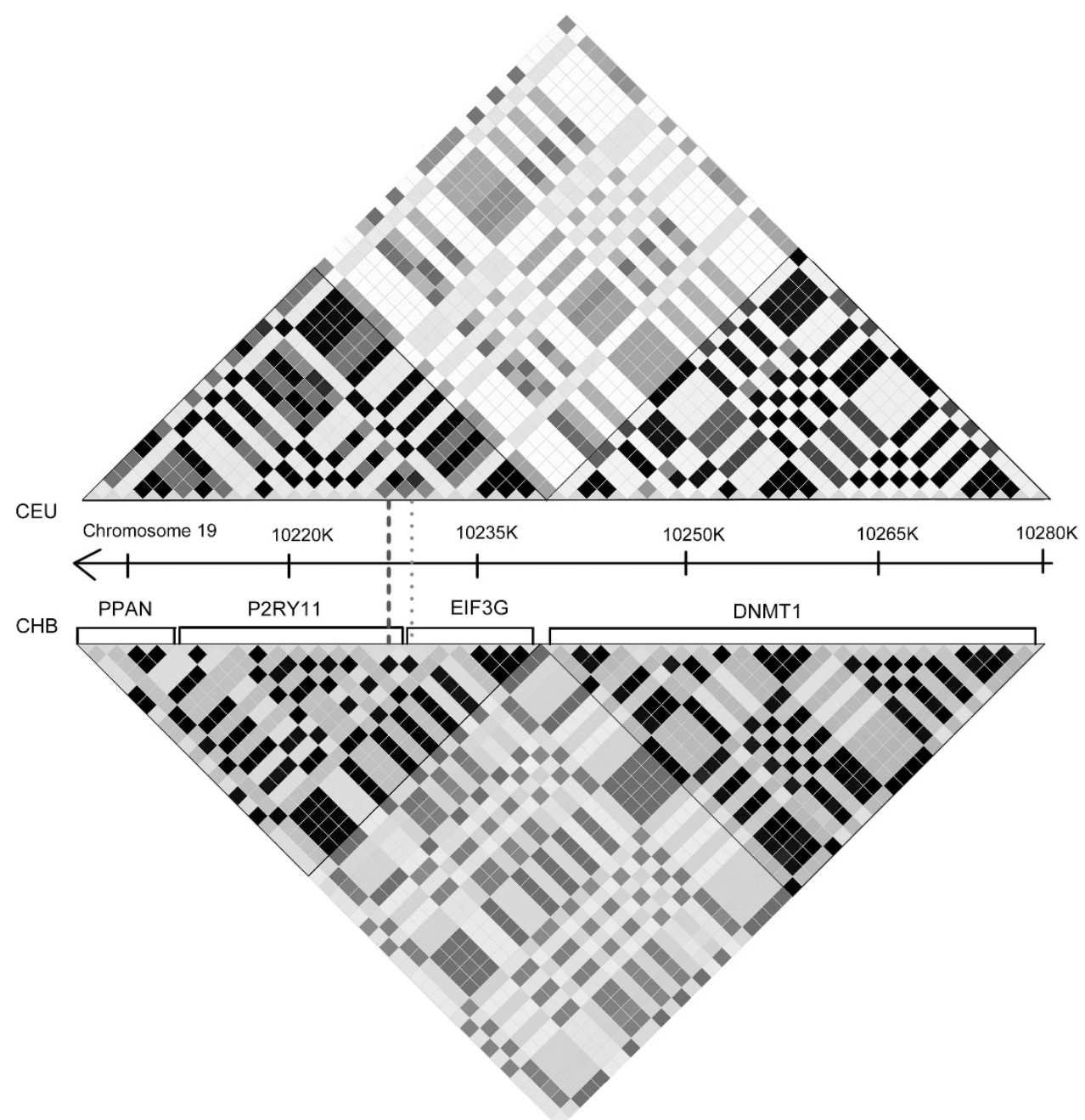

Figure 1 Risk locus on Chr. 19q13.2. Gene organization and LD in the region of interest (10209865-10276 167) compared between European (CEU) and Chinese ancestry (CHB). Top: $R^{2}$-based LD plots using data from GWA data set of European ancestry (CEU). Bottom: $R^{2}$-based LD plots using data from GWA data set of Chinese ancestry (CHB). Darker colors illustrate higher levels of LD. Dashed and dotted line indicates the position of rs2305795 and rs3826784, respectively.
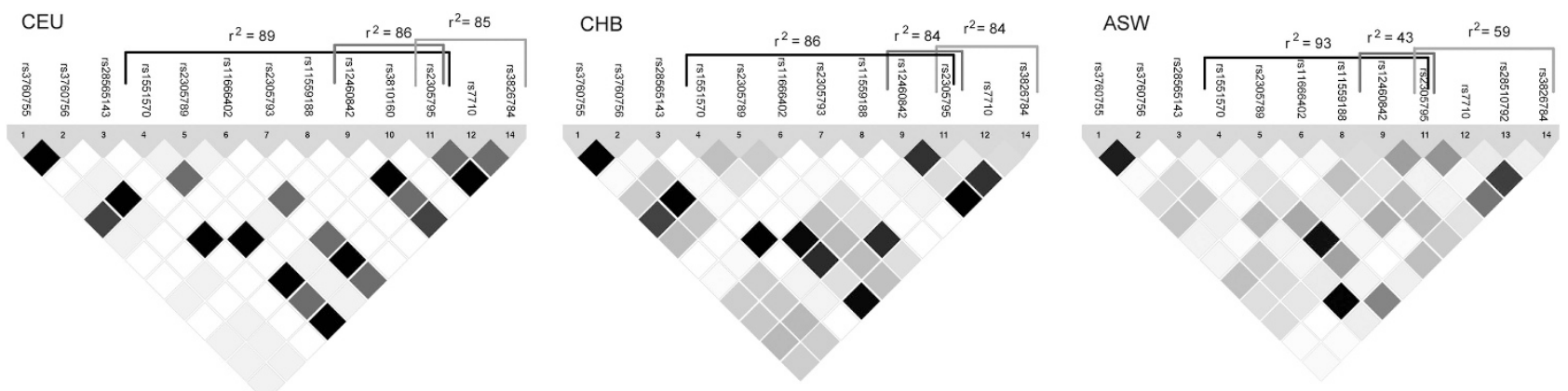

Figure $2 R^{2}$-based LD plots for individuals of CEU, CHB and ASW. Utah residents with Northern and Western European ancestry from the CEPH collection (CEU), Han Chinese in Beijing, China (CHB) and African ancestry in Southwest USA (ASW). Curly braces indicate LD values between SNP candidates: rs1551570, rs12360842, rs2305795 and rs3826784. Darker color illustrates a higher level of LD. Chromosome 19q13.2 (10 209865-10 230 599). See Figure 1 for entire locus.

DNMT1. PPAN and P2RY11 were found to be expressed at very low levels overall in whole blood cells, which correlate with the expression in the brain (Supplementary Tables S2+S3). Expression of EIF3G correlated with the expression of PPAN in normalized data optimized for detecting trans-eQTLs but not in data optimized for detecting ciseQTLs (for more information see Materials and methods). Finally, the expression level of EIF3G was not correlated with DNMT1 (Table 4). We also did not observe a significant correlation between P2RY11 and 
Table 2 Association of SNP markers with Type 1 narcolepsy

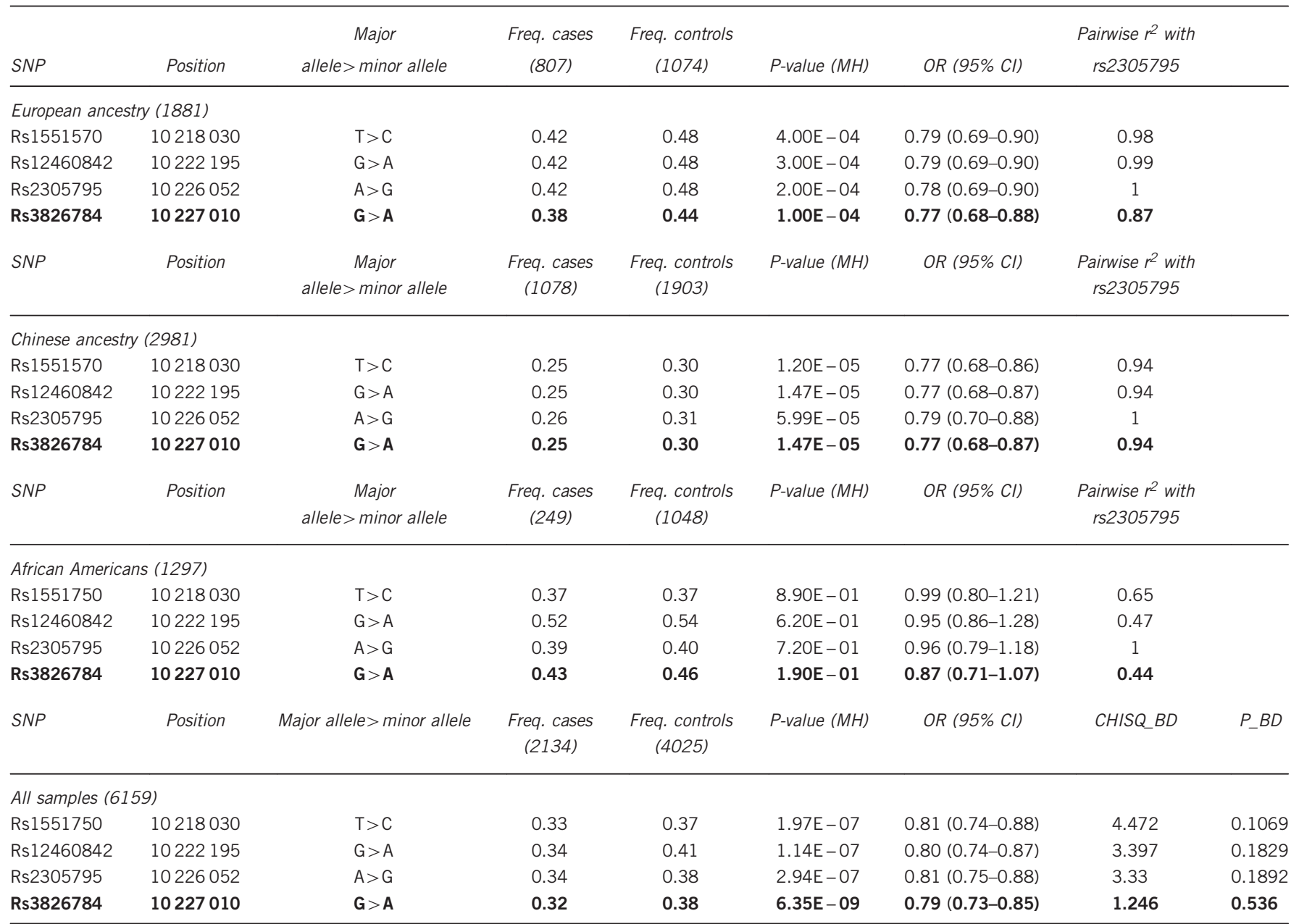

Abbreviations: BD, Breslow day; Cl, confidence interval; Freq., frequency; MH, Mantel-Haenszel; OD, odds ratio; SNP, single-nucleotide polymorphism.

$P$-values were calculated with Beagle. Pairwise LD $\left(R^{2}\right)$ with rs2305795 was calculated with Haploview. Results for minor allele (for European and Chinese ancestry) are presented for each cohort. The selected SNPs were: rs1551570:T>G), rs12460842:A>G), rs2305795:G>A) and rs3826784:A>G). The most significant SNP across all samples is indicated in bold.

PPAN: NM_020230.4; P2RY11: NM_002566.4; EIF3G: NM_003755.3; DNMT1: NM_001130823.1.

DNMT1 in the normalized data for detecting cis-eQTL or trans-eQTL in contrast to what has earlier been reported for PBMCs. ${ }^{5}$

\section{DISCUSSION}

In this study, we found rs3826784, a SNP located within an intron of the EIF3G gene, to be the most significantly associated SNP of the region. Transethnic mapping further revealed that rs3826784 could be a better marker for the genetic association with narcolepsy than the previously identified rs2305795. However, the magnitude of the association is very similar to that of the previously reported SNP rs2305795..$^{5}$ The new SNP, rs3826784, is in high LD with SNPs located in PPAN and P2RY11, but not in DNMT1. Genetic LD between P2RY11 and DNMT1 is weak in narcolepsy and control cohorts, confirming the observation by Han et al. ${ }^{6}$ Importantly, however, elements located within the P2RY11 gene may still regulate DNMT1 expression. We also found that the expression of EIF3G increased with the risk allele, DNMT1 expression decreased and there was no effect of rs $3826784 \mathrm{G}$ on PPAN or P2RY11 mRNA levels in whole blood. The results suggest that the narcolepsy-associated SNP in this locus affects EIF3G expression rather than P2RY11.

To gain insight into gene regulation in the entire risk locus, we investigated gene expression correlations. In whole blood, expression of EIF3G strongly correlated with the expression of PPAN and P2RY11, and less with DNMT1. These results were consistent with strong LD found with SNPs located in the EIF3G, P2RY11 and PPAN region, an effect not extending to DNMT1. Surprisingly, however, expression of P2RY11 and DNMT1 did not correlate as earlier discovered in PBMCs. ${ }^{5}$ PPAN and P2RY11 were found to be expressed at very low levels in whole blood cells. The discrepancy in P2RY11 expression is most likely caused by the different input material in the two studies. Kornum et al. ${ }^{5}$ showed that P2RY11 expression was predominantly seen in $\mathrm{CD}^{+}$cells. These account for $\sim 20 \%$ of PBMCs. However, whole blood also contains granulocytes, which accounts for $65 \%$ of all cells, and may thus mask the expression of P2RY11 in the $\mathrm{CD}^{+}$cells. In agreement with our results, it has previously been reported that EIF3G is highly expressed in blood cells. ${ }^{15}$ These findings illustrate the complexity of interpreting eQTL studies in whole blood, considering heterogeneity of the effect of regulatory regions on gene expression in various white blood cell subsets. Indeed, cell-specific regulatory mechanisms in the PPANP2RY11-EIF3G region are likely present. Future studies will need to address the regulation of the PPAN-P2RY11-EIF3G narcolepsy risk locus within distinct sub-populations of white blood cells. That cellspecific effects are present is not surprising considering the importance 
PPAN

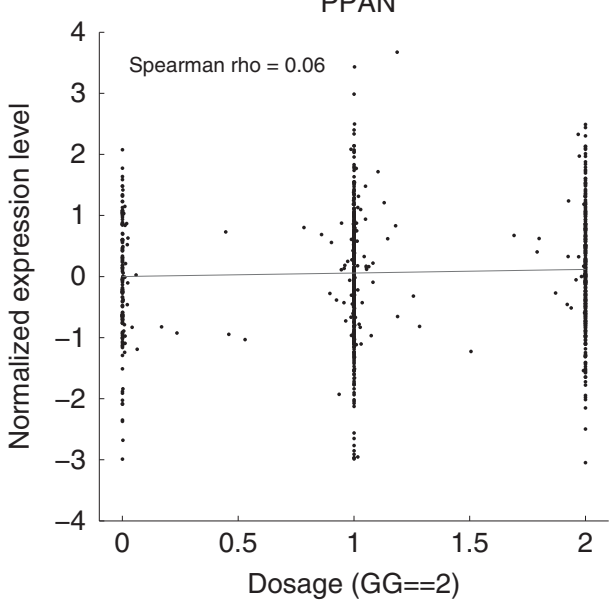

EIF3G

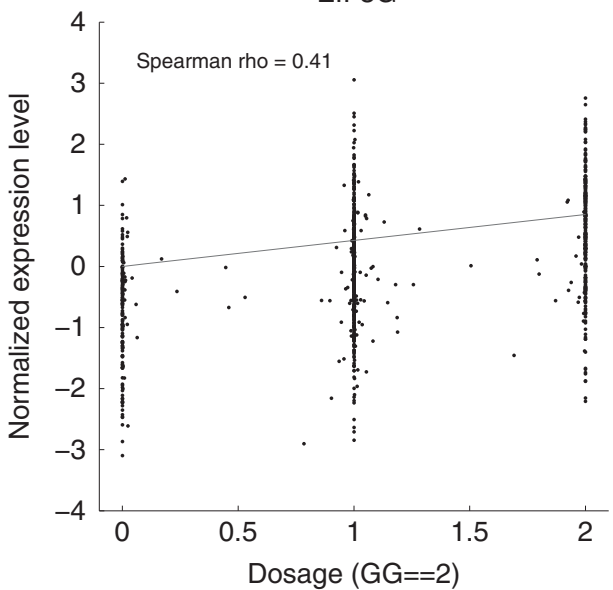

P2RY11

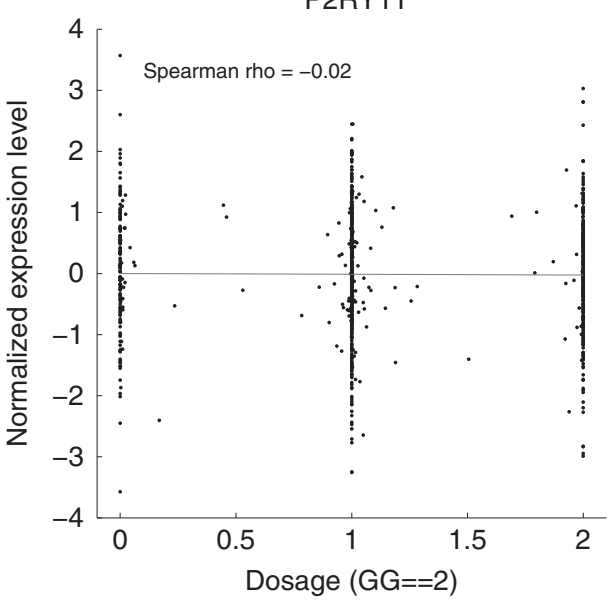

DNMT1

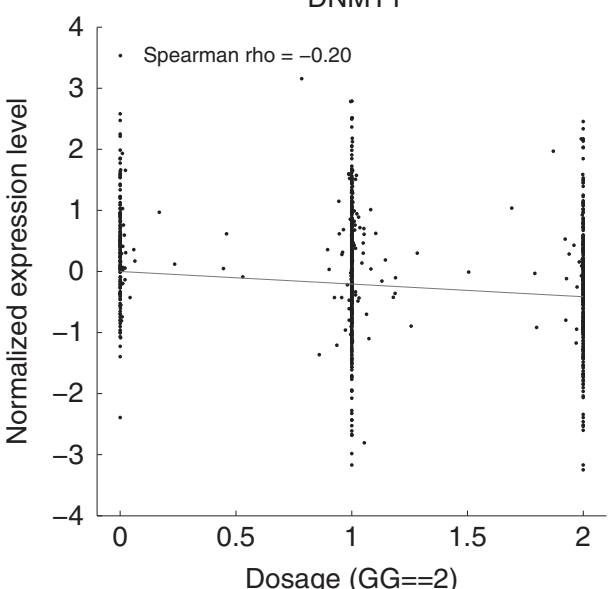

Figure 3 Gene expression in whole blood with various rs3826784 genotypes. (a and $\mathbf{b}$ ) The expression of PPAN and P2RY11 did not change with the risk allele (rs3826784G). (c) The expression of EIF3G increased with the risk allele (rs3826784GG). (d) The expression of DNMT1 decreased with the risk allele (rs3826784GG). The data are reported as normalized effect size, which equals the correlation coefficient (Spearman's $\rho$ correlation coefficient is used as a measure of change in expression level on the y axis).

Table 3 Association between SNPs and eQTLs in the Type 1 narcolepsy risk locus (negative log10 $P$-values for association between genotype and expression levels)

\begin{tabular}{lcccc}
\hline Gene/SNP name & Rs12460842 (genotyped) & Rs2305795 (genotyped) & Rs3826784 (imputed) & Rs1551570 (genotyped) \\
\hline PPAN & 0.94 & 1.01 & 1.10 & 1.00 \\
P2YR11 & 0.14 & 0.22 & 0.27 & 0.18 \\
EIF3G & 42.94 & 43.95 & 38.83 & 43.25 \\
DNMT1 & 10.48 & 10.86 & 9.10 & 10.12 \\
\hline
\end{tabular}

PPAN: NM_020230.4; P2RY11: NM_002566.4; EIF3G: NM_003755.3; DNMT1: NM_001130823.1.

Table 4 Correlation coefficient between gene expression levels of each gene pair was measured using the Spearman's correlation coefficient Correlation coefficient Negative $\log 10$ P-value

\begin{tabular}{|c|c|c|c|c|c|c|c|c|c|}
\hline & PPAN & $P 2 R Y 11$ & EIF3G & DNMT1 & & PPAN & P2RY11 & EIF3G & DNMT1 \\
\hline PPAN & & 0.276 & 0.213 & 0.064 & PPAN & & 16.75 & 10.10 & 1.28 \\
\hline$P 2 R Y 11$ & 0.276 & & 0.155 & 0.008 & $P 2 R Y 11$ & 16.75 & & 5.64 & 0.09 \\
\hline EIF3G & 0.212 & 0.155 & & 0.050 & EIF3G & 10.10 & 5.64 & & 0.88 \\
\hline DNMT1 & 0.064 & 0.008 & 0.050 & & DNMT1 & 1.28 & 0.09 & 0.88 & \\
\hline
\end{tabular}

\footnotetext{
PPAN: NM_020230.4; P2RY11: NM_002566.4; EIF3G: NM_003755.3; DNMT1: NM_001130823.1.
} 
of the regulation of apoptosis in various immune cells types in reaction to changes in the environmental milieu.

How could EIF3G be involved in narcolepsy? Eukaryotic initiation factors (EIF) have an important role in the initiation phase of eukaryotic translation by forming a complex with the $40 \mathrm{~S}$ ribosomal subunit and recruiting a Met-tRNAi called the $43 \mathrm{~S}$ to the preinitiation complex. This complex recognizes and binds the $5^{\prime}$ cap structure of mRNA, promoting ribosomal scanning of mRNA and regulating recognition of the AUG initiation codon. ${ }^{16,17}$ In mammals, EIF3 is the largest scaffolding initiation factor $(750 \mathrm{kDa})$, comprising 13 subunits (A-M). These subunits cluster together in subcomplexes, in which EIF3G connects to EIF3B and EIF3I. EIF3B:G contains RNA recognition motif subunits that are involved in RNA binding. Interestingly, EIF3B:G:I also interacts directly with certain viral mRNAs to promote the translation of viral proteins. ${ }^{18}$ EIF4G, which interacts with EIF3, ${ }^{19}$ contains a binding domain for the non-structural influenza protein, $\mathrm{NS} 1,{ }^{20}$ and is recruited to the viral mRNA to initiate translation of influenza virus. This is particularly interesting as recent findings have implicated a role of the H1N1 pandemic virus of 2009 as a trigger for narcolepsy development. ${ }^{6}$ It has been suggested that the disruption of EIF pathways in childhood $\mathrm{H} 1 \mathrm{~N} 1 / 09$ influenza increases disease severity, ${ }^{21}$ so polymorphisms in this locus could indirectly change immune responses to influenza and narcolepsy risk.

Another possibility could involve regulation of cell death, a function that could be shared with P2RY11 and DNMT1, with coordinated regulation of these loci explaining the conservation of synteny. The $\mathrm{N}$ terminus of EIF3G indeed interacts with the C-terminal region of apoptosis-inducing factor (AIF). AIF is a ubiquitous FAD-binding flavoprotein that has an important role in caspase-independent apoptosis. Mature AIF inhibits newly translated protein synthesis via its interactions with EIF3G. Additionally, mature AIF overexpression specifically activates caspase-7, thereby amplifying the inhibition of protein synthesis including EIF3G cleavage. ${ }^{22,23}$ Additionally, EIF3G is expressed in the brain and the lateral hypothalamus (Allen Brain Atlas: www.brain-map.org). ${ }^{15}$ Higher EIF3G expression with the narcolepsy risk allele could cause a dysregulated cell death, which again could have an important role in white blood cells in the context of autoimmunity, or in the brain in the context of hypocretin cell loss.

In conclusion, using transethnic mapping, we found a novel genetic association of Type 1 narcolepsy with rs3826784, an SNP located in EIF3G. The new SNP is in high LD with the previously reported P2YR11 rs2305795 SNP in individuals of European and Chinese ancestry, but displays lower LD in African Americans. As the association signal from rs3826784 is nominally better compared with rs2305795 in all three cohorts, rs3826784 may be a better marker for the genetic association with narcolepsy, although this conclusion is tentative considering sample size of the African-American Cohort. Further confirmation is needed through extended GWA studies that combine multiple ethnic groups, and local resequencing. A complex correlation between the expression of three genes in the region suggests that a shared regulatory mechanism exists and might be affected by the polymorphism.

\section{CONFLICT OF INTEREST}

The authors declare no conflict of interest.

\section{ACKNOWLEDGEMENTS}

We are indebted to all the narcoleptic patients of the study. Without their contributions this study would not have been possible. This work was supported by the Lundbeck Foundation and NIH Grant P50 NS23724.

1 Matsuki K, Grumet FC, Lin X et al: DQ (rather than DR) gene marks susceptibility to narcolepsy. Lancet 1992;339:1052.

2 Hallmayer J, Faraco J, Lin L et al: Narcolepsy is strongly associated with the T-cell receptor alpha locus. Nat Genet 2009; 41: 708-711.

3 Faraco J, Lin L, Kornum BR et al: ImmunoChip study implicates antigen presentation to T cells in narcolepsy. PLoS Genet 2013; 9: e1003270.

4 Partinen M, Kornum BR, Plazzi G et al: Narcolepsy as an autoimmune disease: the role of H1N1 infection and vaccination. Lancet Neurol 2014; 13: 600-613.

5 Kornum BR, Kawashima M, Faraco J et al: Common variants in P2RY11 are associated with narcolepsy. Nat Genet 2011; 43: 66-71.

6 Han F, Faraco J, Dong XS et al: Genome wide analysis of narcolepsy in China implicates novel immune loci and reveals changes in association prior to versus after the 2009 H1N1 influenza pandemic. PLoS Genet 2013; 9: e1003880.

7 Winkelmann J, Lin L, Schormair B et al: Mutations in DNMT1 cause autosomal dominant cerebellar ataxia, deafness and narcolepsy. Hum Mol Genet 2012; 21 2205-2210.

8 Mignot E, Lin L, Rogers W et al: Complex HLA-DR and -DQ interactions confer risk of narcolepsy-cataplexy in three ethnic groups. Am J Hum Genet 2001; 68: 686-699.

9 Wu Y, Waite LL, Jackson AU et al: Trans-ethnic fine-mapping of lipid loci identifies population-specific signals and allelic heterogeneity that increases the trait variance explained. PLoS Genet 2013; 9: e1003379.

10 Browning SR, Browning BL: Rapid and accurate haplotype phasing and missing data inference for whole genome association studies by use of localized haplotype clustering. Am J Hum Genet 2007; 81: 1084-1097.

11 Barrett JC, Fry B, Maller J et al: Haploview: analysis and visualization of LD and haplotype maps. Bioinformatics 2005; 21: 263-265.

12 Battle A, Mostafavi S, Zhu X et al: Characterizing the genetic basis of transcriptome diversity through RNA-sequencing of 922 individuals. Genome Res 2014; 24: $14-24$.

13 Purcell S, Neale B, Todd-Brown K et al: PLINK: a toolset for whole-genome association and population-based linkage analysis. Am J Hum Genet 2007; 81: 559-575.

14 Stegle O, Parts L, Durbin R, Winn J: A Bayesian framework to account for complex nongenetic factors in gene expression levels greatly increases power in eQTL studies. PLoS Comput Biol 2010; 6: e1000770.

15 Wu C, Orozco C, Boyer J et al: BiogPS: an extensible and customizable portal for querying and organizing gene annotation resources. Genome Biol 2009; 10: R130.

16 Zhou M, Sandercock AM, Fraser CS et al: Mass spectrometry reveals modularity and a complete subunit interaction map of the eukaryotic translation factor elF3. Proc Natl Acad Sci USA 2008; 105: 18139-18144.

17 Hinnebusch AG: elF3: a versatile scaffold for translation initiation complexes. Trends Biochem Sci 2006; 31: 553-562.

18 Park HS, Himmelbach A, Browning KS, Hohn T, Ryabova LA: A plant viral 'reinitiation' factor interacts with the host translational machinery. Cell 2001; 106: 723-733.

19 Spahn CM, Kieft JS, Grassucci RA et al Hepatitis C virus IRES RNA-induced changes in the conformation of the $40 \mathrm{~s}$ ribosomal subunit. Science 2001;291:1959-1962.

20 Aragón T, de la Luna S, Novoa I, Carrasco L, Ortín J, Nieto A: Eukaryotic translation initiation factor $4 \mathrm{GI}$ is a cellular target for NS1 protein, a translational activator of influenza virus. Mol Cell Biol 2000; 20: 6259-6268.

21 Herberg JA, Kaforou M, Gormley S et al: Transcriptomic profiling in childhood H1N1/09 influenza reveals reduced expression of protein synthesis genes. J Infect Dis 2013; 208: 1664-1668.

$22 \mathrm{Kim}$ JT, Kim KD, Song EY et al: Apoptosis-inducing factor (AIF) inhibits protein synthesis by interacting with the eukaryotic translation initiation factor 3 subunit p44 (elF3g). FEBS Lett 2006; 580: 6375-6383.

$23 \mathrm{Kim}$ JT, Lee SJ, Kim BY et al: Caspase-mediated cleavage and DNase activity of the translation initiation factor 3, subunit G (elF3g). FEBS Lett 2013; 587 3668-3674.

Supplementary Information accompanies this paper on European Journal of Human Genetics website (http://www.nature.com/ejhg) 